\title{
Specific Left Ventricular Twist-Untwist Mechanics during Exercise in Children
}

\author{
Julien Boissière, PhD, Claire Maufrais, MS, Georges Baquet, PhD, Iris Schuster, MD, PhD, \\ Michel Dauzat, MD, PhD, Grégory Doucende, PhD, Philippe Obert, PhD, Serge Berthoin, PhD, \\ and Stéphane Nottin, PhD, Lille, Avignon, and Montpellier, France
}

\begin{abstract}
Background: In adults, left ventricular (LV) systolic twist is an important factor that determines LV filling, both at rest and during exercise. In children, lower LV twist has been demonstrated at rest, but its adaptation during exercise and its functional consequences on LV filling are unknown.
\end{abstract}

Methods: Using speckle-tracking echocardiography, LV twist-untwist mechanics were studied in 25 children (aged 10-12 years) and 20 young adults (aged 18-44 years) at rest and during three exercise workloads performed at $20 \%, 30 \%$, and $40 \%$ of their maximal aerobic power.

Results: At rest, LV twist was lower in children, because of a higher temporal dispersion of peak rotation between base and apex. During exercise, the increase of basal rotation was blunted in children compared with adults $\left(-6.7 \pm 2.7^{\circ}\right.$ vs $-9.0 \pm 2.0^{\circ}$ at $40 \%$ of maximal aerobic power, $\left.P<.05\right)$. Consequently, LV twist increased to a lesser extent $\left(13.0 \pm 5.0^{\circ}\right.$ vs $15.8 \pm 4.5^{\circ}$ at $40 \%$ of maximal aerobic power, $\left.P<.05\right)$. The increase in LV untwisting rates during exercise was also lower in children, leading to a lower percentage of untwisting during early diastole ( $8 \pm 8 \%$ vs $29 \pm 20 \%$ at $40 \%$ of maximal aerobic power, $P<.001)$. Consequently, during early diastole, the normal timing of diastolic events observed in young adults, with untwist occurring before radial displacement, was blunted in children. Nevertheless, children exhibited normal LV filling due to higher diastolic radial and longitudinal strain rates.

Conclusions: Twist-untwist mechanics may evolve with advancing age. In children, early diastolic LV untwisting appears to be less important than in adults. Their better LV intrinsic myocardial relaxation may ensure adequate LV filling during exercise without dependence on the additional effect of suction resulting from LV energy recoil. (J Am Soc Echocardiogr 2013;26:1298-305.)

Keywords: Children, Left ventricle, Speckle-tracking echocardiography, Exercise

Because of the helical orientation of the myofibers, ${ }^{1,2}$ cardiomyocyte shortening during systole induces not only left ventricular (LV) normal strains but also shear strains. LV twist is one of these shear strains, characterized by simultaneous basal clockwise and apical counterclockwise rotation. LV untwisting occurs very early in diastole (i.e., predominantly during isovolumic relaxation), thus promoting LV suction by increasing the intraventricular pressure gradient from the apex to the base. ${ }^{3}$ During exercise, the increase in heart rate dramatically shortens diastole duration, and a recent

From EA 4488-Activité Physique-Muscle-Santé, Université Lille Nord de France, Faculté des Sciences du Sport et de l'Education Physique, Lille, France (J.B., G.B., S.B.); LAPEC EA 4278, F-8400, Avignon University, Avignon, France (C.M. G.D., P.O., S.N.); and EA 2992-Dysfonctions des Interfaces Vasculaires, Faculté de Médecine, Université Montpellier 1, Montpellier, France (I.S., M.D.).

Reprint requests: Stéphane Nottin, PhD, EA 4278 - Laboratoire de PharmÉcologie Cardiovasculaire, Université d'Avignon et des Pays de Vaucluse, Faculté des Sciences, 33 Rue Louis Pasteur, 84000 Avignon, France (E-mail: stephane. nottin@univ-avignon.fr).

0894-7317/\$36.00

Copyright 2013 by the American Society of Echocardiography.

http://dx.doi.org/10.1016/j.echo.2013.07.007

1298 study demonstrated a major increase in LV untwisting resulting from an increase in both systolic basal and apical rotation. ${ }^{4}$ This "systolic-diastolic" coupling via the LV twist-untwist sequence constitutes one key element maintaining LV filling and stroke volume during exercise in healthy individuals. ${ }^{4-6}$

It was recently proposed that LV twist mechanics were age related. ${ }^{5,7,8}$ Aging is accompanied by increased LV twist at rest, ${ }^{9-11}$ which limits its ability to increase during exercise. ${ }^{9}$ In children, both basal and apical LV rotation and thus LV twist were lower at rest compared with young adults. Moreover, children exhibit a greater diastolic untwisting rate and a greater amount of untwisting during isovolumic relaxation., 10,12 Thereby, these specific "LV twist mechanics" in children may contribute to different responses to exercise than those observed in adults. Yet data regarding the kinetics of LV twist and untwist mechanics during exercise in children are still lacking.

We used speckle-tracking echocardiography (STE) to assess kinetics of LV myocardial mechanical events in children at rest and during an incremental exercise test. STE constitutes a reliable and suitable method to study ventricular myocardial function at rest ${ }^{13,14}$ and during exercise $e^{4,6,9,15}$ and is suitable for pediatric investigations. 7,12 We hypothesized that the time course of LV twisting and untwisting mechanics during exercise will be altered in children because of 
Abbreviations

AVC = Aortic valve closure
IVRT = Isovolumetric
relaxation time
LV = Left ventricular
SR = Strain rate
STE = Speckle-tracking
echocardiography
${ }^{\text {UT }}$ IVRT = Untwisting angle
during isovolumetric
relaxation time
W1 = Exercise at $20 \%$ of
maximal aerobic power
W2 = Exercise at $30 \%$ of
maximal aerobic power
W3 = Exercise at $40 \%$ of
maximal aerobic power

years). None reported regular training habits or had any clinical or anamnestic evidence of cardiovascular disease or arterial hypertension. Subjects were excluded if resting echocardiography demonstrated an ejection fraction $<50 \%$, significant valvular disease, or abnormal right ventricular function. Boys were examined clinically, and their pubertal status was assessed using Tanner stages. All boys were at Tanner stage 1 . This study received approval from the local ethics committee, and written informed consent was obtained from the adults and from all children and their parents. The study conformed to the latest revision of the Declaration of Helsinki.

\section{Experimental Protocol}

Body height and mass were assessed. For both children and adults, maximal aerobic power was initially estimated via the Wasserman equation, \{body mass $\times[(50.72-0.372 \times$ age $)]-350\} / 10.3$, and corrected for the semisupine position $(20 \%$ were deduced from calculated values). For children, the estimated maximal aerobic power was checked by an additional incremental exercise protocol (stages of 1 min on a E-Bike ergometer; GE Healthcare, Milwaukee, WI).

For the cardiovascular evaluation, subjects were positioned on the dedicated semisupine cycling ergometer (E-Bike). After a 15-min to 20-min resting period, each subject underwent an incremental exercise test that included three workloads of 6 min at 20\% (W1), 30\% (W2), and 40\% (W3) of maximal aerobic power, followed by incremental workloads of $1 \mathrm{~min}$ until exhaustion. During the test, the pedaling rate was kept constant at 70 to $80 \mathrm{rpm}$ for all subjects.

\section{Echocardiographic Data Acquisition}

Two-dimensional and Doppler echocardiographic data were recorded at the end of the resting period and during the last $4 \mathrm{~min}$ of the W1, W2, and W3 stages, with commercially available systems (Vivid; GE Vingmed Ultrasound AS, Horten, Norway) using a 3.5$\mathrm{MHz}$ sector-scanning electronic transducer (M4S; GE Vingmed Ultrasound AS). All measurements were taken by a single observer who was well experienced in exercise echocardiography. We recorded cine loops in parasternal short-axis (basal and apical levels) and apical four-chamber views during a 2 -sec to 3-sec breath-holding period at the end of breathing out. Two-dimensional grayscale harmonic images were obtained at a rate of 65 to 90 frames/sec. Images were acquired in cine loops triggered by the QRS complex and saved for offline analysis, which was performed using dedicated software (EchoPAC version 6.0; GE Vingmed Ultrasound AS) Systemic arterial blood pressure was measured at rest and during each stage of exercise in the left arm using manual sphygmomanometry and auscultation. During the last $30 \mathrm{sec}$ of each workload, we measured aortic blood flow velocity in the ascending aorta with a 2.0-MHz continuous-wave Doppler transducer (Pedof P2D CW; GE Vingmed Ultrasound) placed at the suprasternal notch for stroke volume determination, as previously described. ${ }^{17,18}$

\section{Data Analysis}

M-mode measurements were obtained offline from the parasternal short-axis view. LV inflow $\mathrm{E}$ and $\mathrm{A}$ waves were recorded using pulsed Doppler in the apical four-chamber view. From electrocardiographic recordings, we measured the time delays from the onset of the QRS complex to the onset of aortic blood flow (aortic valve opening delay) to the end of aortic blood flow (aortic valve closure [AVC] delay), to the onset of early filling blood flow (mitral valve opening delay), and to the peak of early filling blood flow (peak E). Isovolumetric relaxation time (IVRT) was calculated as mitral valve opening minus AVC.

Speckle-tracking analysis of LV strain and twist was conducted as previously described. ${ }^{4,19,20}$ After manually tracing the endocardial border on the end-systolic frame of the $2 \mathrm{D}$ sequence, the software automatically tracked myocardial motion in this region of interest Whenever the software signaled poor tracking efficiency, the observer readjusted the endocardial trace line and/or the width of the region of interest until a satisfying tracking score was obtained. All measurements were averaged over three to five cardiac cycles. LV longitudinal strain and strain rate (SR) were assessed in an apical four-chamber view. Radial strain, circumferential strain and SR, and LV rotation and rotational rate were assessed from short-axis views at the basal and apical levels. Particular care was taken to ensure that the basal short-axis plane contained the mitral valve and that the apical plane was acquired with the transducer in a caudal position (i.e., below the papillary muscles) to improve LV apical rotation measurement. $^{8}$

Two-dimensional strain and SR data were processed using a specific toolbox developed in our laboratory with Scilab version 4.1 (Scilab; INRIA, Le Chesnay, France). For temporal analysis, this software ad justed all strain and SR variables for intersubject differences in heart rate and transducer frame rate acquisition. The time sequence was normalized by interpolation to the percentage of systolic duration (i.e., AVC represented $100 \%$ of systole). After normalization, the soft ware averaged the data from three to five cardiac cycles and performed the detection of peak strain and SR events and their timing (expressed as a percentage of systolic duration). Net LV twist was calculated as the instantaneous difference between LV apical and basal rotation. The following indices of diastolic function were calculated during IVRT: the untwisting angle $\left({ }^{\circ} \mathrm{UT}_{\text {IVRT }}=\right.$ twist at AVC - twist at the end of IVRT), the percentage of untwisting during IVRT $\left({ }^{\circ} \mathrm{UT}_{\text {IVRT }} /\right.$ [twist at $\left.\left.\mathrm{AVC}\right] \times 100\right)$, and the mean untwisting rate during IVRT ( $\left.{ }^{\circ} \mathrm{UT}_{\text {IVRT }} / \mathrm{IVRT}\right)$. To assess the dynamics of global LV twist and its coupling with LV mean radial displacement (reflecting volumetric changes of the left ventricle) throughout the cardiac cycle (systole and diastole), graphical loops were built using time-aligned values of LV twist and radial displacement throughout the cardiac cycle. Mean radial displacement values were averaged from six segments obtained in basal and apical short-axis planes. 


\section{Statistical Analysis}

Values are expressed as mean \pm SD in tables and text and as mean \pm $\mathrm{SE}$ in figures. Statistical analysis was performed using StatView version 5.0 (SAS Institute Inc., Cary, NC). For comparison of subject characteristics, unpaired $t$ tests were performed. For cardiac variables, oneway analysis of variance (i.e., age group) with repeated measures (i.e., resting or exercise intensities) was performed with post hoc Bonferroni correction as appropriate. Linear regressions were used to determine the relationships between LV twist and LV untwisting rate during exercise. Statistical significance for all analyses was considered at $P<.05$. Intraobserver reproducibility of speckle-tracking echocardiographic indices has been assessed previously in our laboratory on 12 subjects, and coefficients of variation were $<8 \%$ for both strain and rotation. ${ }^{20}$

\section{RESULTS}

Subjects' characteristics at rest are presented in Table 1. Briefly, children showed lower height and mass than adults $(P<.05)$. Conventional morphologic echocardiographic measurements showed lower LV mass relative to body surface area as well as lower LV relative wall thickness $(P<.05)$, suggesting more compliant ventricles in children for the latter parameter.

During exercise, the W1, W2, and W3 stages were performed at similar relative intensities (percentages of maximal aerobic power) in both groups, that is, at $19 \pm 1 \%, 28 \pm 1 \%$, and $38 \pm 2 \%$ of $\max -$ imal aerobic power in adults and $18 \pm 3 \%, 28 \pm 4 \%$, and $37 \pm 5 \%$ in children $(P>.05)$. Nevertheless, heart rates were higher in children at each stage: $112 \pm 9,118 \pm 9$, and $128 \pm 10$ beats $/ \mathrm{min}$ in children versus $100 \pm 12,110 \pm 12$, and $121 \pm 12$ beats $/ \mathrm{min}$ in adults $(P<.05)$. In both groups, normalized stroke volume increased significantly from rest to $\mathrm{W} 2$ and remained constant between $\mathrm{W} 2$ and W3. At maximal exercise (Table 2), mechanical power and mean arterial pressure were significantly lower in children, whereas maximal relative oxygen uptake was significantly higher $(P<.05)$. Nevertheless, indexed cardiac output and estimated LV filling pressure (E/e' ratio) were similar in the two groups $(P>.05)$

In both groups, longitudinal strain increased during exercise (from $-18.0 \pm 3.0 \%$ at rest to $-20.4 \pm 2.6 \%$ at $\mathrm{W} 3$ in children vs from $-17.6 \pm 2.1 \%$ to $-23.2 \pm 1.9 \%$ in adults, $P<.05)$. However, longitudinal diastolic SR was higher in children at each stage (from $2.05 \pm$ $0.33 \mathrm{sec}^{-1}$ at rest to $2.46 \pm 0.40 \mathrm{sec}^{-1}$ at W3 in children vs from 1.41 \pm 0.34 to $2.22 \pm 0.38 \mathrm{sec}^{-1}$ in adults, $P<.05$ ). Similar results were obtained for radial diastolic SR (from $-2.48 \pm 0.57 \mathrm{sec}^{-1}$ at rest to $-3.49 \pm 0.79 \mathrm{sec}^{-1}$ at W3 in children vs from $-1.72 \pm 0.33$ to $-2.79 \pm 0.49 \mathrm{sec}^{-1}$ in adults, $P<.05$ ).

\section{Systolic Rotation and Twist during Exercise}

Kinetics of peak LV basal and apical rotation and twist from rest to W3 are presented in Figure 1, and values at rest and at W3 are presented in Table 3. During exercise, apical rotation increased progressively in both groups. However, the increase in basal rotation was higher in adults, and LV twist was therefore lower in children at each workload. Kinetics of time to peak rotation and twist expressed as a percentage of systolic duration are presented in Figure 2. From rest to maximal exercise, peak basal rotation was delayed in children. Peak apical rotation occurred earlier in children, but only at rest and at W1. Consequently, the delay between time to peak basal and apical rotation was higher in children at rest and at W1.
Table 1 Subject characteristics

\begin{tabular}{lcc}
\hline \multicolumn{1}{c}{ Variable } & $\begin{array}{c}\text { Children } \\
(n=25)\end{array}$ & $\begin{array}{c}\text { Adults } \\
(n=20)\end{array}$ \\
\hline Age $(\mathrm{y})$ & $11 \pm 1$ & $25 \pm 9^{*}$ \\
Height $(\mathrm{cm})$ & $144 \pm 7$ & $177 \pm 5^{\star}$ \\
Weight $(\mathrm{kg})$ & $37 \pm 8$ & $72 \pm 8^{*}$ \\
BSA $\left(\mathrm{m}^{2}\right)$ & $1.22 \pm 0.13$ & $1.88 \pm 0.12^{*}$ \\
Resting mean arterial pressure $(\mathrm{mm} \mathrm{Hg})$ & $76 \pm 5$ & $99 \pm 7^{*}$ \\
LV mass/BSA $\left(\mathrm{g} / \mathrm{m}^{2}\right)$ & $53.8 \pm 9.5$ & $92.5 \pm 14.4^{*}$ \\
Relative wall thickness & 0.25 & $0.35 \pm 0.05^{\star}$ \\
\hline
\end{tabular}

$B S A$, Body surface area.

Data are expressed as mean \pm SD.

${ }^{\star} P<.001$ versus children.

Table 2 Maximal exercise parameters

\begin{tabular}{|c|c|c|}
\hline Variable & $\begin{array}{l}\text { Children } \\
(n=25)\end{array}$ & $\begin{array}{l}\text { Adults } \\
(n=20)\end{array}$ \\
\hline Maximal aerobic power (W) & $114 \pm 26$ & $221 \pm 33^{\dagger}$ \\
\hline $\begin{array}{l}\text { Maximal oxygen uptake } \\
(\mathrm{mL} / \mathrm{min} / \mathrm{kg})\end{array}$ & $45.7 \pm 9.3$ & $36.0 \pm 5.7^{\star}$ \\
\hline $\begin{array}{l}\text { Indexed maximal cardiac output } \\
\left(\left\llcorner/ \mathrm{min} / \mathrm{m}^{2}\right)\right.\end{array}$ & $11.1 \pm 3.0$ & $10.9 \pm 1.9$ \\
\hline $\begin{array}{l}\text { Maximal mean arterial pressure } \\
(\mathrm{mm} \mathrm{Hg})\end{array}$ & $92 \pm 9$ & $128 \pm 9^{\dagger}$ \\
\hline E/e' ratio & $9.0 \pm 1.7$ & $8.8 \pm 1.3$ \\
\hline
\end{tabular}

Data are expressed as mean \pm SD.

${ }^{\star} P<.01$ and ${ }^{\dagger} P<.001$ versus children.

\section{Diastolic Untwist during Exercise}

In both groups, significant $(P<.05)$ and similar correlations (Figure 3$)$ were found between LV systolic twist and untwisting rate $\left(R^{2}=0.43\right.$ in children vs $R^{2}=0.50$ in adults, $P>.05$ ) from rest to W3. Kinetics of LV twist and twisting rate as well as basal and apical rotation at rest and at W3 are presented in Figure 4. During exercise, the increase in peak LV untwisting rate was lower in children than in adults. Therefore, peak untwisting rate was lower at W3 in children (Figure 4). LV diastolic variables are presented in Table 4 . Time to peak untwisting rate did not significantly change during exercise in children or in adults. There was no significant change in ${ }^{\circ} \mathrm{UT}_{\mathrm{IVRT}}$ during exercise, but it was significantly lower in children. Mean untwisting rate during IVRT remained constant in children, whereas it increased during exercise in adults. The percentage of untwisting during IVRT decreased during exercise in both groups, but children showed lower values than adults.

Building of twist-radial displacement variation loops (Figure 5) demonstrated different patterns during the cardiac cycle in children and adults. Both at rest and during exercise, systole was characterized by a roughly linear relationship between LV twist and radial displacement in the two groups (the "ejection phase" in Figure 5), that is, a parallel increase between LV twist and LV diameter decreasing. However, during diastole (the "relaxation phase" in Figure 5), adults exhibited at first rapid untwisting (before mitral opening), followed by LV radial expansion. In children, this specific timing of mechanical events (uncoupling between LV untwist and radial expansion) was not observed. Indeed, the relaxation phase was characterized by better coupling between LV untwisting and radial expansion in this group. 

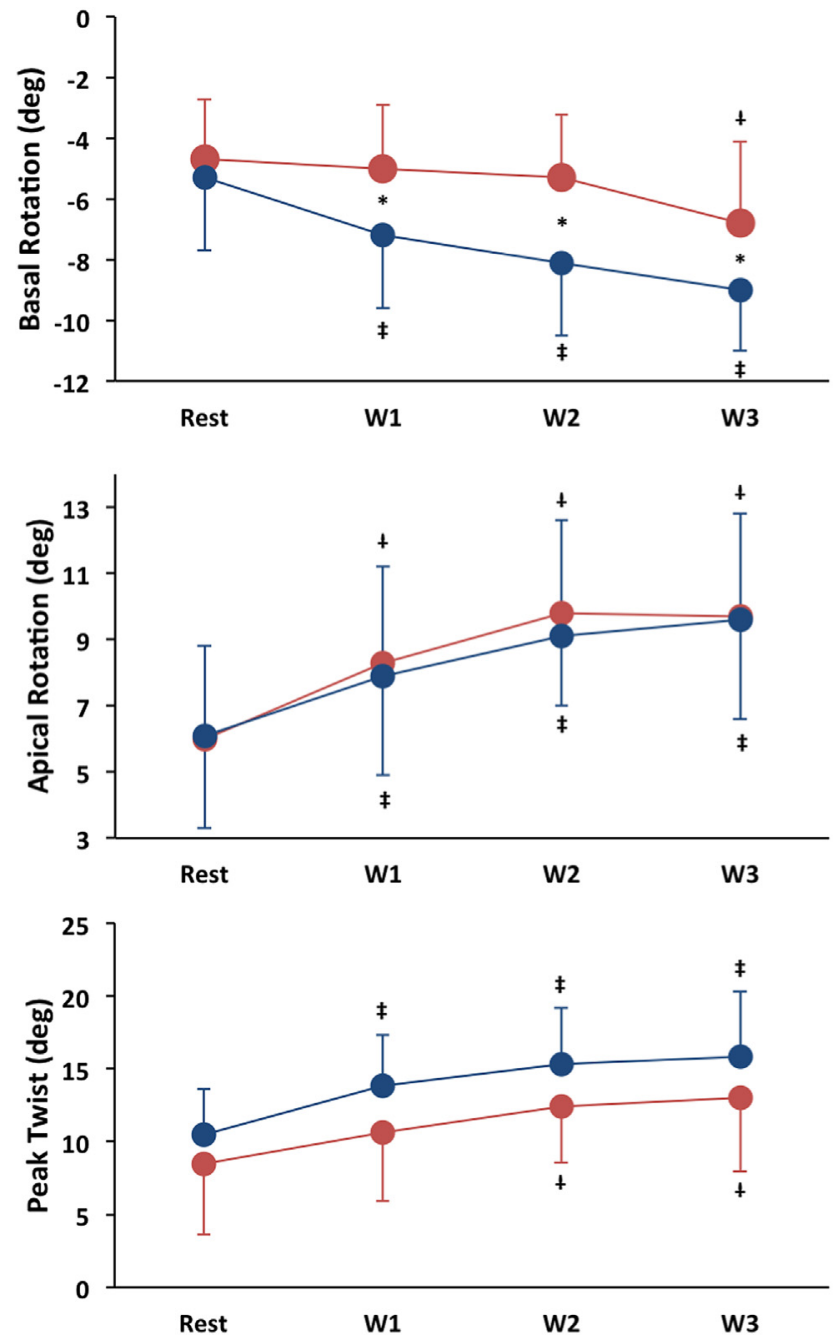

Figure 1 Kinetics of basal (top) and apical (middle) rotation and corresponding LV twist (bottom) from rest to W3 in adults (blue lines and circles) and children (red lines and circles). *Significant difference from children. ${ }^{\dagger}$ Significant difference from resting conditions in children. ${ }^{\ddagger}$ Significant difference from resting conditions in adults.

\section{DISCUSSION}

The major finding of this study was that children present specific LV twist-untwist mechanics during exercise. As observed in adults, LV systolic twist increased progressively with exercise intensity in children but remained lower. LV untwisting in early diastole was also lower, and the uncoupling between LV early untwisting and radial expansion was partially abolished in children. Nevertheless, children had normal LV diastolic function during exercise because of their higher LV relaxation properties and compliance.

\section{Specific LV Rotation and Twist in Children during Exercise}

Under resting conditions, LV twist was lower in children than in adults. Previous studies have reported an increase in resting LV twist with aging. ${ }^{7}, 10,12,21$ In neonates, LV basal rotation is counterclockwise and then becomes progressively clockwise with age.,10 With growth, clockwise basal rotation becomes more prominent, resulting in an increase in net LV twist. ${ }^{7,10,12}$ These mechanical changes have been explained by the increase of the radii of epicardial layers in the developing heart. However, in the present study, similar basal and apical rotation was observed in children and in adults. Interestingly, the lower LV twist observed in children was concomitant with a higher apical-to-basal peak rotation delay due to both earlier apical and late basal rotations. Because LV twist is the instantaneous result of basal and apical peak rotations, this increased delay could be responsible for the lower LV twist in children. The timing of basal and apical rotations has never been assessed in children, but previous studies have shown a decrease of apical-to-basal temporal dispersion with aging. $^{7,22,23}$ The higher apical-to-basal temporal dispersion in children could be due to a specific apical-to-basal electric activation sequence in the left ventricle, ${ }^{24}$ but this remains to be confirmed.

During exercise, LV twist increased regularly in children, a pattern also recently observed in adults. ${ }^{4-6}$ Nevertheless, an important result of the present study was that LV twist remained significantly lower in children at each workload during exercise. Of note, during submaximal exercise at W2 and W3, the greater apical-to-basal temporal dispersion observed at rest in children was totally abolished. The lower twist in children resulted only from a lower increase in basal rotation during exercise, because there was no difference in apical rotation between children and adults. Interestingly, the counterclockwise basal rotation observed in early systole during exercise was higher in children and could have contributed in part to their lower basal rotation (Figure 4, bottom). During early systole, the brief basal counterclockwise rotation is due to the mechanical activity of the subendocardial fibers. ${ }^{24,25}$ With aging, early basal counterclockwise rotation decreases concomitantly with the functional decline of the subendocardial fibers. ${ }^{8,26}$ On the other hand, lower LV twist was also observed in endurance-trained athletes, ${ }^{27,28}$ in whom specific endocardial adaptations were suggested. Hence, the higher counterclockwise basal rotation and the lower LV twist observed in our pediatric population could also be due to better endocardial function compared with adults. ${ }^{11,26}$

\section{Specific LV Untwist Mechanics in Children during Exercise}

In adults, LV diastolic untwisting, a consequence of both the relaxation of the myocardium and the release of the energy stored in compressed titin and elastic components, occurs very early in diastole and promotes LV "suction" by increasing the LV intraventricular (i.e., from apex to base) pressure gradient. ${ }^{3,29}$ During incremental exercise, whereas diastolic duration dramatically shortens, LV untwisting rate progressively increases concomitantly with enhanced systolic torsion (i.e., there is a linear relationship between LV twist and untwisting rate). ${ }^{4}$ In children, this "systolic-diastolic" relationship is also present, as LV twist and untwisting rate were tightly correlated (Figure 3). However, the latter was lower in children during exercise, especially at W3. These age-related differences could be linked to the lower LV systolic twist observed in children as previously described. ${ }^{5}$ Some authors have also suggested the switch from titin isoform N2A to isoform N2B as an explanation for these differences. ${ }^{30}$ Indeed, the amount of the N2B isoform is greater in adults and allows higher restoring forces during early diastole.

In adults, a large proportion of LV diastolic untwisting occurs in early diastole (i.e., during IVRT). The amount of untwisting during the isovolumic period accounts for LV filling efficiency because it generates an apical-to-basal pressure gradient. ${ }^{3}$ In the present study, mean untwisting rate during IVRT failed to increase in children compared with adults, and consequently, the percentage of untwisting during IVRT dramatically fell in children during exercise. As a result, 
Table 3 LV rotation and twisting parameters

\begin{tabular}{|c|c|c|c|c|}
\hline \multirow[b]{2}{*}{ Variable } & \multicolumn{2}{|c|}{ Rest } & \multicolumn{2}{|c|}{ Exercise } \\
\hline & Children & Adults & Children & Adults \\
\hline \multicolumn{5}{|l|}{ Apical rotation } \\
\hline Peak $\left(^{\circ}\right)$ & $5.9 \pm 2.7$ & $6.1 \pm 2.7$ & $9.6 \pm 3.2^{\S}$ & $9.6 \pm 3.2^{\S}$ \\
\hline Time to peak (\%) & $71.6 \pm 18.9$ & $91.4 \pm 6.9^{\dagger}$ & $74.4 \pm 21.3$ & $72.8 \pm 18.4^{\ddagger}$ \\
\hline \multicolumn{5}{|l|}{ Basal rotation } \\
\hline Peak $\left(^{\circ}\right)$ & $-5.0 \pm 1.9$ & $-5.3 \pm 2.4$ & $-6.7 \pm 2.7^{\S}$ & $-9.0 \pm 2.0^{\dagger, \S}$ \\
\hline Time to peak (\%) & $120.4 \pm 23.2$ & $108.0 \pm 20.2^{\star}$ & $117.3 \pm 22.7$ & $111.2 \pm 17.2^{\star}$ \\
\hline \multicolumn{5}{|l|}{ Twist } \\
\hline Peak $\left(^{\circ}\right)$ & $8.5 \pm 4.9$ & $10.4 \pm 3.2^{\dagger}$ & $13.0 \pm 5.0^{\S}$ & $15.8 \pm 4.5^{\dagger, \S}$ \\
\hline Time to peak (\%) & $97.1 \pm 6.4$ & $92.4 \pm 4.5$ & $94.8 \pm 9.8$ & $91.5 \pm 6.0$ \\
\hline
\end{tabular}

Data are expressed as mean \pm SD.

${ }^{\star} P<.05$ and ${ }^{\dagger} P<01$. versus children.

${ }^{\ddagger} P<.01$ and ${ }^{\S} P<.001$ versus resting conditions.
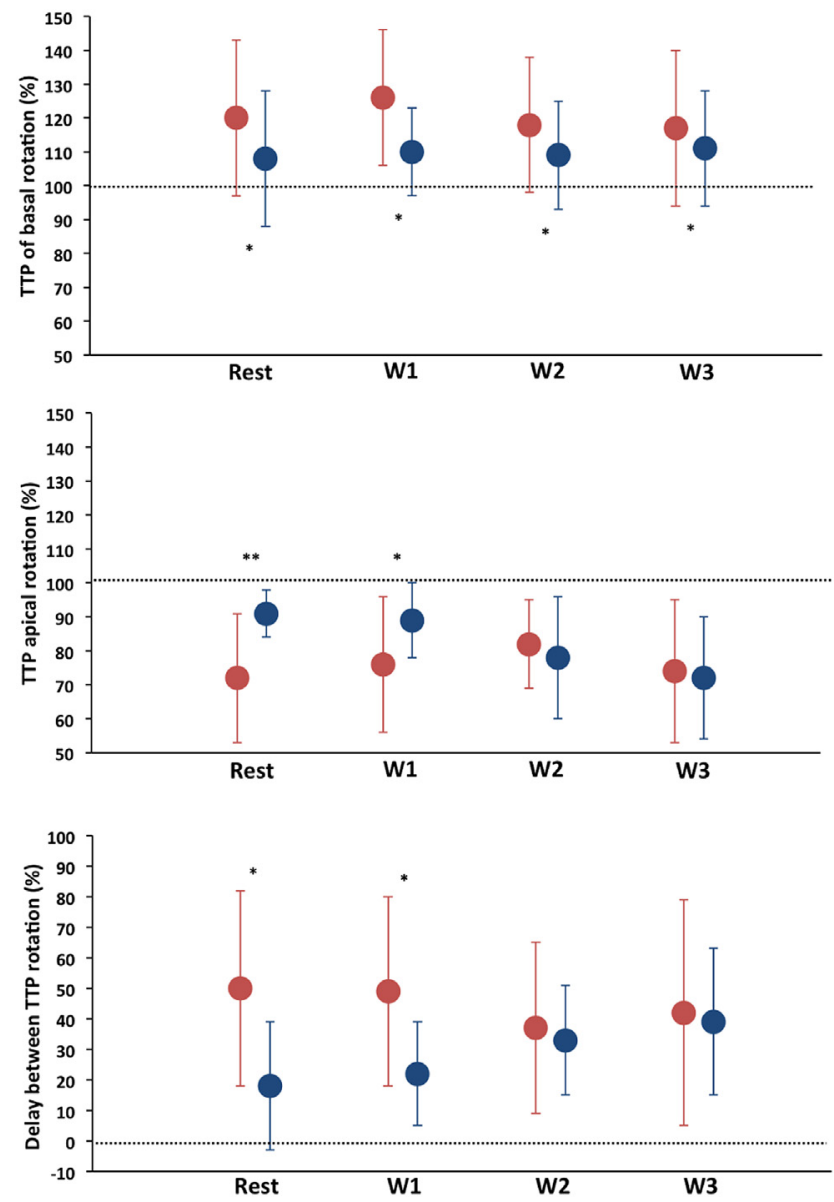

Figure 2 Kinetics of basal (top) and apical (middle) time to peak (TTP) rotation and the corresponding delay between apical and basal TTP rotation (bottom) from rest to workload W3 in adults (blue circles) and in children (red circles). *Significant difference from children.

the twist-radial displacement loops exhibited specific patterns in children. In adults, LV untwisting preceded the normal strains that drive LV filling (i.e., radial and longitudinal LV expansion) at rest and during exercise. In children, this specific timing was not observed. All in all,

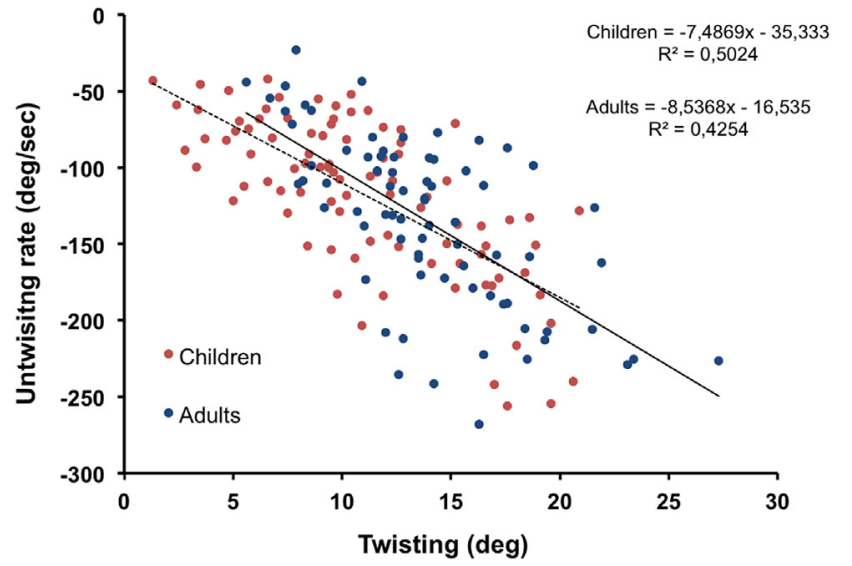

Figure 3 Linear regression between LV peak twist and peak untwisting rate from W1 to W3 in adults (blue circles) and children (red circles), with regression lines for adults (solid line) and children (dotted line).

these blunted untwisting mechanics raise the question of their efficiency in diastolic filling during exercise in children.

Despite the fall in LV diastolic recoil during IVRT observed in children, global early diastolic performance during exercise, as evidenced by the transmitral $\mathrm{E}$ wave, was similar than those observed in adults. This suggests, in children, that LV filling was less dependent on elastic restoring forces and that other underlying mechanisms, such as LV relaxation and/or compliance, could be involved. In animal models, it was demonstrated that calcium reuptake by the sarcoplasmic reticulum was faster in young adult than in mature rats, suggesting higher myocardial intrinsic relaxation properties. ${ }^{31,32}$ In our study, indices of sarcomere relengthening velocity, such as diastolic longitudinal SR on STE, ${ }^{33,34}$ were higher in children. Thus, greater LV relaxation properties in children, as suggested by higher longitudinal and radial diastolic SR, could account for their normal early filling despite a lower LV untwisting rate. In addition, similar LV filling pressures $\left(\mathrm{E} / \mathrm{e}^{\prime}\right.$ ratios) between children and adults at each stage of workload reinforce the hypothesis of better myocardial relaxation rather than a ventricular preload effect. Nevertheless, LV compliance could also account for diastolic LV filling. We could not exclude an effect of this property on the specific twist-untwist mechanics observed in children, as we observed lower relative LV wall thickness (an indicator of 

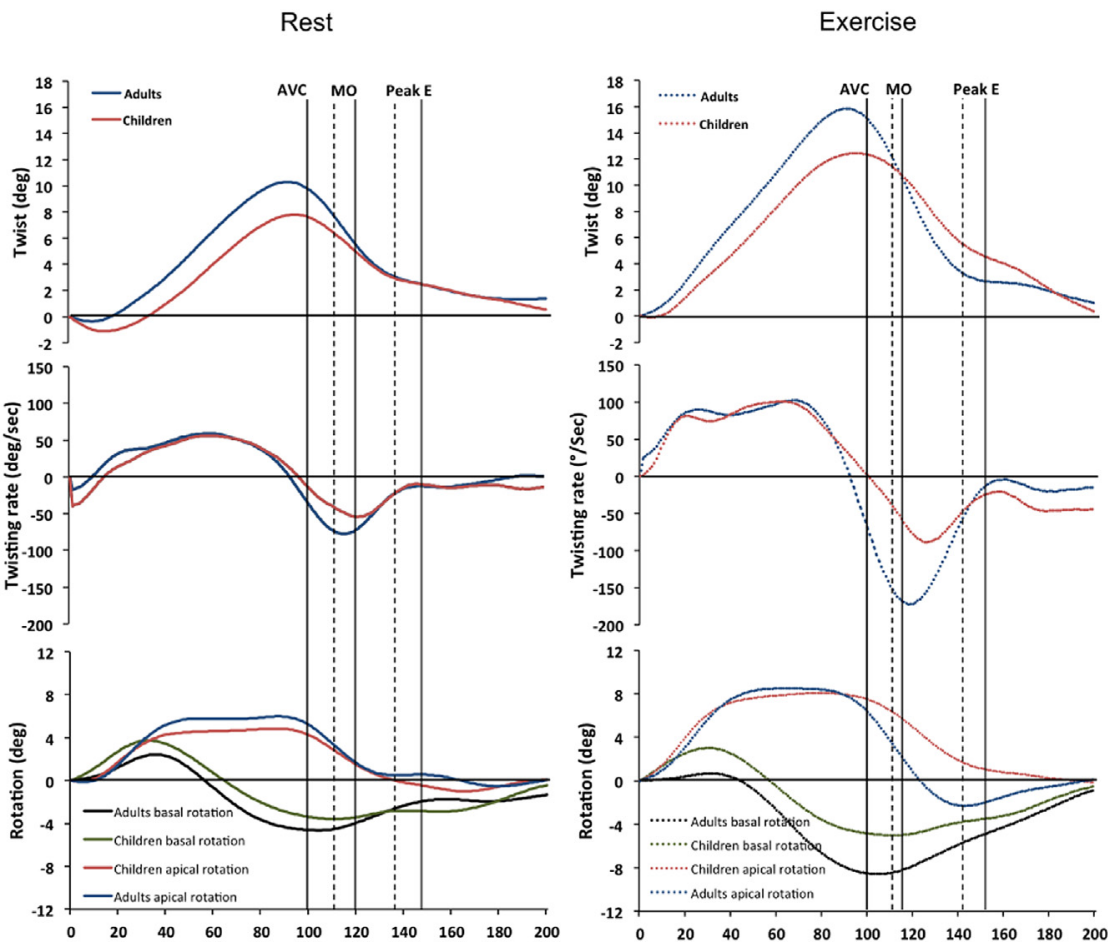

Figure 4 Pattern of LV twist-untwist (top), twisting-untwisting rates (middle), and basal-apical rotation (bottom) during the cardiac cycle averaged in 20 adults and 25 children at rest (left) and at W3 (right). Values are expressed as percentages of systolic duration in adults and in children, with green and black lines showing basal rotation values in children and adults, respectively, and red and blue lines showing apical rotation in children and adults, respectively. $M O$, Mitral valve opening.

Table 4 LV diastolic parameters

\begin{tabular}{|c|c|c|c|c|}
\hline \multirow[b]{2}{*}{ Variable } & \multicolumn{2}{|c|}{ Rest } & \multicolumn{2}{|c|}{ w3 } \\
\hline & Children & Adults & Children & Adults \\
\hline Diastolic duration (msec) & $522 \pm 113$ & $608 \pm 128^{*}$ & $230 \pm 30$ & $272 \pm 31^{\star, \S}$ \\
\hline IVRT (msec) & $41.2 \pm 13.7$ & $65.6 \pm 18.0$ & $26.1 \pm 10.8$ & $35.1 \pm 10.2$ \\
\hline Systolic time duration (\%) & $13.3 \pm 4.5$ & $20.3 \pm 5.6^{\dagger}$ & $11.9 \pm 5.1^{\ddagger}$ & $15.7 \pm 4.9^{\dagger, \ddagger}$ \\
\hline \multicolumn{5}{|l|}{ Mitral valve opening } \\
\hline Systolic time duration (\%) & $113.3 \pm 4.5$ & $120.4 \pm 5.6^{\dagger}$ & $111.9 \pm 5.1^{\ddagger}$ & $115.7 \pm 4.9^{\dagger, \S}$ \\
\hline \multicolumn{5}{|l|}{ Peak E } \\
\hline Peak $(\mathrm{cm} / \mathrm{sec})$ & $96 \pm 12$ & $88 \pm 19$ & $128 \pm 17^{\S}$ & $126 \pm 17^{\S}$ \\
\hline Systolic time duration (\%) & $138.4 \pm 5.1$ & $148.6 \pm 9.0^{\dagger}$ & $142.8 \pm 7.7$ & $151.2 \pm 10.4^{\dagger}$ \\
\hline \multicolumn{5}{|l|}{ Untwisting rate } \\
\hline Peak $(\% / \mathrm{sec})$ & $-78.7 \pm 33.8$ & $-88.7 \pm 34.3$ & $-140.5 \pm 51.7^{\S}$ & $182.9 \pm 53.5^{\star, \S}$ \\
\hline Time to peak (\%) & $113.3 \pm 44.7$ & $117.4 \pm 6.7$ & $132.0 \pm 38.9$ & $121.9 \pm 7.2$ \\
\hline${ }^{\circ} \mathrm{UT}_{\text {IVRT }}\left({ }^{\circ}\right)$ & $1.4 \pm 1.1$ & $4.0 \pm 1.5^{\dagger}$ & $1.0 \pm 1.1$ & $3.9 \pm 1.9^{\dagger}$ \\
\hline Mean untwisting rate during IVRT $\left({ }^{\circ} / \mathrm{sec}\right)$ & $34.3 \pm 29.5$ & $64.1 \pm 28.9^{*}$ & $38.3 \pm 37.7$ & $110.7 \pm 47.6^{\dagger, \S}$ \\
\hline Percentage of untwisting during IVRT & $25.7 \pm 28.6$ & $45.3 \pm 22.5^{\dagger}$ & $8.8 \pm 9.2^{\S}$ & $29.8 \pm 20.4^{\dagger, \S}$ \\
\hline
\end{tabular}

Data are expressed as mean \pm SD.

${ }^{\star} P<.01$ and ${ }^{\dagger} P<.001$ versus children.

${ }^{\ddagger} P<.01$ and ${ }^{\S} P<.001$ versus resting conditions.

ventricular compliance) in children. Nevertheless, further studies are needed to confirm this hypothesis. The increase in LV twist and subsequent untwist observed in adults could thus be interpreted as a compensatory mechanism that appears with the decrease in ventricular relaxation and compliance properties with aging. This hypothesis is supported by the fact that an increase in LV twist is also observed in pathologic conditions associated with diastolic dysfunction, such as hypertrophic cardiomyopathy. ${ }^{35}$

\section{Study Limitations}

We should address several limitations with regard to the present study. Because of technical limitations, four adults and three children recruited for the study were excluded because of inadequate speckletracking data. In this study, children were all recruited at the same school and were only 10 or 11 years old, which was not a true representation of the overall pediatric population. Indeed, it has been 

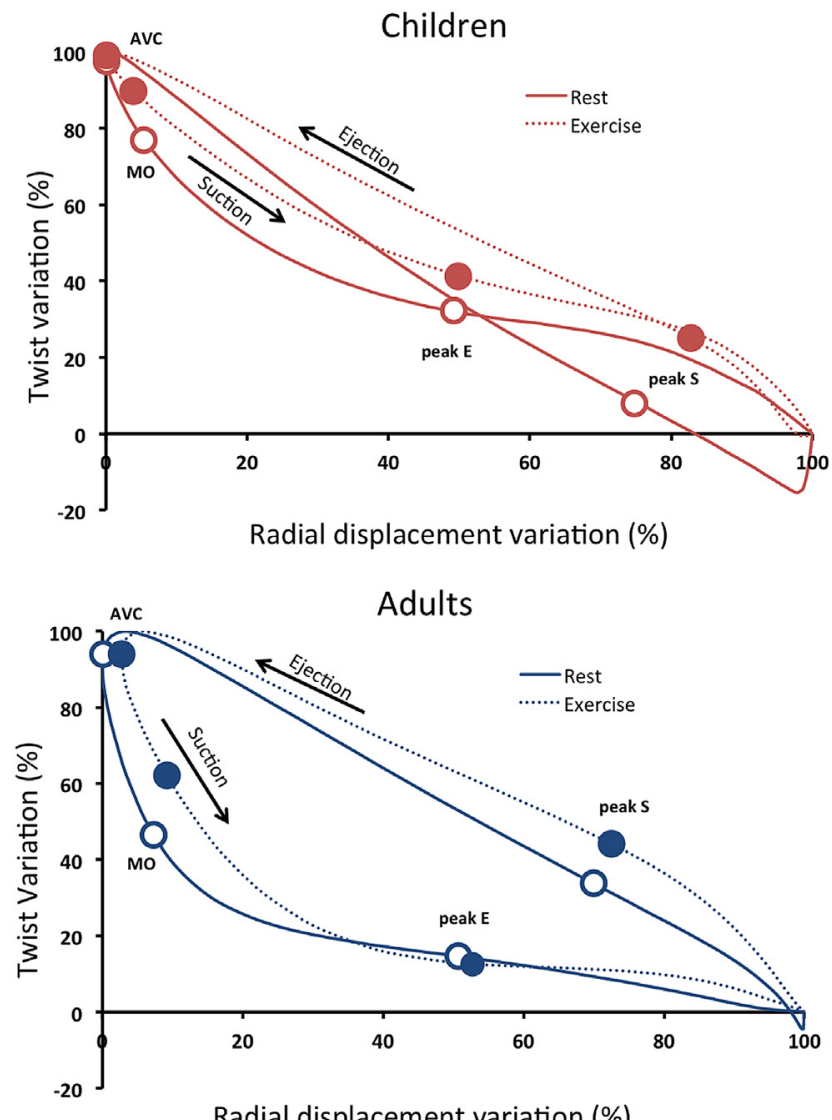

Radial displacement variation (\%)

Figure 5 Twist-radial displacement variation loops in children and adults at rest (red and blue dotted lines, respectively) and during exercise (W3; red and blue solid lines, respectively).

demonstrated that $\mathrm{LV}$ twist and untwisting velocities vary from the infant period to adolescence. ${ }^{10,22}$ Limited frame rates during STE allow only submaximal exercise intensities (i.e., $120-130$ beats/min at W3). Hence, the data presented here should be extrapolated with caution to exercise performed at high intensities. In addition, diastolic filling is a complex interplay among preload, afterload, and myocardial function on both the left atrium and the left ventricle. ${ }^{36}$ The influence of atrial function on twist and untwist mechanics and the subsequent LV filling cannot be excluded as a factor for alterations observed in children. Indeed, the influence of atrial function on LV filling has been demonstrated after prolonged exercise $\mathrm{e}^{37}$ and in athlete's heart. ${ }^{38}$

\section{CONCLUSIONS}

This study provides new insights into LV mechanics in children during exercise. Our results strongly support the existence of specific LV twist and untwist patterns in children and raise the question of the involvement of $\mathrm{LV}$ untwisting in improving diastolic function during exercise in children who have excellent relaxation properties and compliance.

\section{REFERENCES}

1. Ingels NB Jr., Hansen DE, Daughters GT II, Stinson EB, Alderman EL, Miller DC. Relation between longitudinal, circumferential, and oblique shortening and torsional deformation in the left ventricle of the transplanted human heart. Circ Res 1989;64:915-27.
2. Streeter DD Jr., Spotnitz HM, Patel DP, Ross J Jr., Sonnenblick EH. Fiber orientation in the canine left ventricle during diastole and systole. Circ Res 1969;24:339-47.

3. Notomi Y, Popovic ZB, Yamada H, Wallick DW, Martin MG, Oryszak SI, et al. Ventricular untwisting: a temporal link between left ventricular relax ation and suction. Am J Physiol Heart Circ Physiol 2008;294:H505-13.

4. Doucende G, Schuster I, Rupp T, Startun A, Dauzat M, Obert P, et al. Kinetics of left ventricular strains and torsion during incremental exercise in healthy subjects: the key role of torsional mechanics for systolic-diastolic coupling. Circ Cardiovasc Imaging 2010;3:586-94.

5. Notomi Y, Martin-Miklovic MG, Oryszak SJ, Shiota T, Deserranno D, Popovic ZB, et al. Enhanced ventricular untwisting during exercise: a mechanistic manifestation of elastic recoil described by Doppler tissue imaging. Circulation 2006;113:2524-33.

6. Stohr EJ, Gonzalez-Alonso J, Shave R. Left ventricular mechanical limitations to stroke volume in healthy humans during incremental exercise Am J Physiology Heart Circ Physiol 2011;301:H478-87.

7. Takahashi K, Al Naami G, Thompson R, Inage A, Mackie AS, Smallhorn JF. Normal rotational, torsion and untwisting data in children, adolescents and young adults. J Am Soc Echocardiogr 2010;23:286-93.

8. van Dalen BM, Vletter WB, Soliman OI, ten Cate FJ, Geleijnse ML. Importance of transducer position in the assessment of apical rotation by speckle tracking echocardiography. J Am Soc Echocardiogr 2008;21:895-8.

9. Burns AT, La Gerche A, MacIsaac AI, Prior DL. Augmentation of left ventricular torsion with exercise is attenuated with age. J Am Soc Echocardiogr 2008;21:315-20.

10. Notomi Y, Srinath G, Shiota T, Martin-Miklovic MG, Beachler L, Howell K, et al. Maturational and adaptive modulation of left ventricular torsional biomechanics: Doppler tissue imaging observation from infancy to adulthood. Circulation 2006;113:2534-41.

11. Takeuchi M, Nakai H, Kokumai M, Nishikage T, Otani S, Lang RM. Agerelated changes in left ventricular twist assessed by two-dimensional speckle-tracking imaging. J Am Soc Echocardiogr 2006; 19:1077-84.

12. Zhang Y, Zhou QC, Pu DR, Zou L, Tan Y. Differences in left ventricular twist related to age: speckle tracking echocardiographic data for healthy volunteers from neonate to age 70 years. Echocardiography 2010;27:1205-10.

13. Amundsen BH, Helle-Valle T, Edvardsen T, Torp H, Crosby I, Lyseggen E, et al. Noninvasive myocardial strain measurement by speckle tracking echocardiography: validation against sonomicrometry and tagged magnetic resonance imaging. J Am Coll Cardiol 2006;47:789-93.

14. Korinek J, Wang J, Sengupta PP, Miyazaki C, Kjaergaard J, McMahon E, et al. Two-dimensional strain-a Doppler-independent ultrasound method for quantitation of regional deformation: validation in vitro and in vivo. I Am Soc Echocardiogr 2005; 18:1247-53.

15. Esch BT, Scott JM, Warburton DE, Thompson R, Taylor D, Cheng Baron J et al. Left ventricular torsion and untwisting during exercise in heart transplant recipients. J Physiol 2009;587:2375-86.

16. Nottin S, Vinet A, Stecken F, Nguyen LD, Ounissi F, Lecoq AM, et al. Central and peripheral cardiovascular adaptations during a maximal cycle exercise in boys and men. Med Sci Sports Exerc 2002;34:456-63.

17. Schuster I, Thoni G), Ederhy S, Walther G, Nottin S, Vinet A, et al. Subclinical cardiac abnormalities in human immunodeficiency virus-infected men receiving antiretroviral therapy. Am J Cardiol 2008;101:1213-7.

18. Vinet A, Nottin S, Lecoq AM, Guenon P, Obert P. Reproducibility of cardiac output measurements by Doppler echocardiography in prepubertal children and adults. Int J Sports Med 2001;22:437-41.

19. Notomi Y, Lysyansky P, Setser RM, Shiota T, Popovic ZB, Martin Miklovic MG, et al. Measurement of ventricular torsion by twodimensional ultrasound speckle tracking imaging. J Am Coll Cardiol 2005;45:2034-41.

20. Nottin S, Doucende G, Schuster I, Tanguy S, Dauzat M, Obert P. Alteration in left ventricular strains and torsional mechanics after ultralong duration exercise in athletes. Circ Cardiovasc Imaging 2009;2:323-30.

21. Oxenham HC, Young AA, Cowan BR, Gentles TL, Occleshaw CI, Fonseca $C G$, et al. Age-related changes in myocardial relaxation using three-dimensional tagged magnetic resonance imaging. J Cardiovasc Magn Reson 2003;5:421-30. 
22. Al-Naami GH. Torsion of young hearts: a speckle tracking study of normal infants, children, and adolescents. Eur J Echocardiogr 2010;11:853-62.

23. van Dalen BM, Soliman OI, Vletter WB, ten Cate FJ, Geleijnse ML. Agerelated changes in the biomechanics of left ventricular twist measured by speckle tracking echocardiography. Am J Physiol Heart Circ Physiol 2008; 295:H1705-11.

24. Sengupta PP, Korinek J, Belohlavek M, Narula J, Vannan MA, Jahangir A, et al. Left ventricular structure and function: basic science for cardiac im aging. J Am Coll Cardiol 2006;48:1988-2001.

25. Buckberg GD, Castella M, Gharib M, Saleh S. Structure/function interface with sequential shortening of basal and apical components of the myocardial band. Eur J Cardiothorac Surg 2006;29(suppl):S75-97.

26. Lumens I, Delhaas T, Arts T, Cowan BR, Young AA. Impaired subendocardial contractile myofiber function in asymptomatic aged humans, as de tected using MRI. Am J Physiol Heart Circ Physiol 2006;291:H1573-9.

27. Nottin S, Doucende G, Schuster-Beck I, Dauzat M, Obert P. Alteration in left ventricular normal and shear strains evaluated by $2 \mathrm{D}$-strain echocardiography in the athlete's heart. I Physiol 2008;586:4721-33.

28. Zocalo Y, Bia D, Armentano RL, Arias L, Lopez C, Etchart C, et al. Assessment of training-dependent changes in the left ventricle torsion dynamics of professional soccer players using speckle-tracking echocardiography. IEEE Eng Med Biol Soc Conf Proc 2007;2007:2709-12.

29. Rademakers FE, Buchalter MB, Rogers WJ, Zerhouni EA, Weisfeldt ML, Weiss JL, et al. Dissociation between left ventricular untwisting and filling. Accentuation by catecholamines. Circulation 1992;85:1572-81.

30. Lahmers S, Wu Y, Call DR, Labeit S, Granzier H. Developmental control of titin isoform expression and passive stiffness in fetal and neonatal myocardium. Circ Res 2004;94:505-13.
31. Howlett SE. Age-associated changes in excitation-contraction coupling are more prominent in ventricular myocytes from male rats than in myocytes from female rats. Am J Physiol Heart Circ Physiol 2010;298:H659-70.

32. Kandilci HB, Tuncay E, Zeydanli EN, Sozmen NN, Turan B. Age-related regulation of excitation-contraction coupling in rat heart. J Physiol Biochem $2011 ; 67: 317-30$.

33. Hashimoto I, Li X, Hejmadi Bhat A, Jones M, Zetts AD, Sahn DJ. Myocardial strain rate is a superior method for evaluation of left ventricular subendocardial function compared with tissue Doppler imaging. J Am Coll Cardiol 2003;42:1574-83.

34. Wang J, Khoury DS, Thohan V, Torre-Amione G, Nagueh SF. Global diastolic strain rate for the assessment of left ventricular relaxation and filling pressures. Circulation 2007;115:1376-83.

35. Soullier C, Obert P, Doucende G, Nottin S, Cade S, Perrez-Martin A, et al Exercise response in hypertrophic cardiomyopathy: blunted left ventricular deformational and twisting reserve with altered systolic-diastolic coupling. Circ Cardiovasc Imaging 2012;5:324-32.

36. Abhayaratna WP, Seward JB, Appleton CP, Douglas PS, Oh JK, Tajik AJ, et al. Left atrial size: physiologic determinants and clinical applications. J Am Coll Cardiol 2006;47:2357-63.

37. Oxborough D, Whyte G, Wilson M, O'Hanlon R, Birch K, Shave R, et al. A depression in left ventricular diastolic filling following prolonged strenuous exercise is associated with changes in left atrial mechanics. J Am Soc Echocardiogr 2010;23:968-76.

38. D’Ascenzi F, Cameli M, Zaca V, Lisi M, Santoro A, Causarano A, et al. Supernormal diastolic function and role of left atrial myocardial deformation analysis by $2 \mathrm{D}$ speckle tracking echocardiography in elite soccer players. Echocardiography $2011 ; 28: 320-6$.

\section{CORRIGENDUM}

In the article entitled "Expert Consensus for Multi-Modality Imaging Evaluation of Cardiovascular Complications of Radiotherapy in Adults: A Report from the European Association of Cardiovascular Imaging and the American Society of Echocardiography" by Lancellotti et al (J Am Soc Echocardiogr 2013;26:1013-32), there was an error in the listing of an author's name. The correct listing is Jutta Bergler-Klein. 